\title{
THE SHEAR MODULUS OF A MATERIAL PERMEATED BY A RANDOM DISTRIBUTION OF FREE CIRCULAR CRACKS*
}

\author{
By H. D. GARBIN* and L. KNOPOFF (University of California, Los Angeles)
}

1. Introduction. In an earlier paper (1), we considered the solutions to the problem: of the propagation of compressional elastic waves through a medium permeated by a random distribution of randomly oriented circular cracks. The cracks are sparsely distributed and have radii small compared with the wavelength.

The method of solution involves the formulation of a set of dual integral equations which represents the scattered field from a single, obliquely-inclined scatterer. One pair of dual integral equations is decoupled and represents the $S H$ field, that is, the field of shear waves polarized parallel to the plane of the circular crack. The other equations are a pair of coupled dual integral equations and represent the coupled $S V$ and $P$ fields, that is, the field of waves polarized perpendicular to the direction of propagation and to $\mathrm{SH}$ and the field of compression waves. The integral equations are, of necessity, inhomogeneous, with the inhomogeneity describing the incident field. The integral equations are inverted by showing that they are in the form of Abel's equation.

The result in [1] was presented in the form of an effective compliance for compressional waves in the composite. To the accuracy of the approximations involved, we could as easily have written the modulus. The result was obtained by a self-consistent procedure; we find the effective compliance of the composite in terms of an integral over the jump in the displacement $\left[u_{i}\right]$ across the faces of the crack and averaged over all possible directions of orientation of the crack. The dominant term in the jump in the displacement perpendicular to the crack surface $\left[u_{2}\right]$ is the monopolar or symmetric term, while that for the radial $\left[u_{r}\right]$ and azimuthal terms $\left[u_{\phi}\right]$ are the dipolar terms. We find that, as a result of taking the average over all directions of cracks, we get the result

$$
\left\langle\frac{1}{\lambda+2 \mu}\right\rangle=\frac{1}{(\lambda+2 \mu)_{0}}\left(1+\frac{8}{3} \frac{N a^{3}}{v} f(\sigma)\right)
$$

where the compliance in the diamond brackets is that for the composite, and $(\lambda+2 \mu)_{0}$ is the modulus of the unflawed material. The compliance of the composite increases as the number of cracks per unit volume $N / v$ increases. This quantity is made nondimensional through the introduction of the cube of the radius of the crack $a^{3}$ as a scaling factor. The crux of the calculation is the determination of the function $f(\sigma)$, where $\sigma$ is Poisson's ratio.

In order to complete the catalogue of properties of a homogeneously flawed material, we must write down the value of a second effective modulus. For seismological reasons, we choose to calculate the effective shear modulus, that is, the apparent modulus of

* Received May 29, 1974. Publication No. 1311, Institute of Geophysics and Planetary Physies, University of California, Los Angeles.

** Present address: Sandia Corporation, Albuquerque, N. M. 
elasticity for the composite when excited by plane shear waves of long wavelength. We may expect that the mathematical development in this problem will be parallel to that of the case of excitation by longitudinal, i.e. compressional, waves. Since the boundary conditions remain the same, while only the nature of the incident waves is changed, we expect that only the inhomogeneous terms of the integral equations will be changed; the homogeneous terms, i.e. the unknown wave amplitudes, will have the same coefficients in both cases.

Since the formal procedures are expected to be identical to [1], we shall only give a sketch of the calculation. We shall give occasional reproductions of the complete equations in order to give the reader who is trying to duplicate the algebraic work some guideposts. We use the same equation numbers as in [1]; as a consequence, the equation numbers in this paper are non-consecutive.

2. The integral equations. The incident field for shear waves is

$$
\begin{gathered}
\Phi^{0}=0, \\
\left(\begin{array}{c}
\chi^{0} \\
\Psi^{0}
\end{array}\right)=\left(\begin{array}{c}
A / k_{2} \\
B
\end{array}\right) \exp (i(\alpha x+\beta z))=\left(\begin{array}{c}
A / k_{2} \\
B
\end{array}\right) \exp (i \beta z) \sum_{n=-\infty}^{\infty} i^{n} \exp (i n \phi) J_{n}(\alpha r), \\
\alpha^{2}+\beta^{2}=k_{2}{ }^{2} .
\end{gathered}
$$

The amplitude of the potential $\chi^{0}$ is divided by the shear wave number $k_{2}$ in order that the quantities $A$ and $B$ be in the same dimensions; reference to Eqs. (4) will establish this condition.

The dual integral equations are

$$
\begin{array}{cc}
\int_{0}^{\infty} \frac{\nu_{2}}{\nu_{1}} A^{\prime}(\zeta) S_{n}(\zeta) J_{n}(\zeta r) d \zeta=\frac{2 \alpha^{2} \beta}{k_{2}{ }^{3}} A i^{n+1} J_{n}(\zeta r), & 0 \leq r \leq 1, \\
\int_{0}^{\infty} S_{n}(\zeta) J_{n}(\zeta r) d \zeta=0, & r>1, \\
\int_{0}^{\infty} \zeta\left[A^{\prime}(\zeta) L_{n}(\zeta)+\nu_{2} Q_{2 n}(\zeta r)\right] J_{n-1}(\zeta) d \zeta & \\
\int_{0}^{\infty} \zeta\left[L_{n}(\zeta)+Q_{2 n}(\zeta)\right] J_{n-1}(\zeta r) d \zeta=0, & \\
\int_{0}^{\infty} \zeta\left[A^{\prime}(\zeta) L_{n}(\zeta)-\nu_{2} Q_{2 n}(\zeta)\right] J_{n+1}(\zeta r) d \zeta & \\
=\left[A\left(k_{2}{ }^{2}-2 \beta^{2}\right) / k_{2}+\beta B\right] i^{n+1} \alpha J_{n+1}(\alpha r), & 0 \leq r \leq 1, \\
\int_{0}^{\infty} \zeta\left[L_{n}(\zeta)-Q_{2 n}(\zeta)\right] J_{n+1}(\zeta r) d \zeta=0, & r>1,
\end{array}
$$

where $A^{\prime}(\zeta)$ is defined in (40).

3. The solution to the dual integral equations. We continue the analogy with [1]. We propose solutions as in (26), to which we add 


$$
\begin{gathered}
\zeta L_{0}(\zeta)=\zeta^{1 / 2} \int_{0}^{\infty} p_{10}(\eta) J_{3 / 2}(\zeta \eta) d \eta \\
\zeta Q_{20}(\zeta)=\zeta^{1 / 2} \int_{0}^{\infty} q_{20}(\eta) J_{3 / 2}(\zeta \eta) d \eta .
\end{gathered}
$$

We substitute (26) into (16), (17) and (18) and invert by means of Abel's solution. We get (36a), (35a), (35b) and

$$
\begin{aligned}
& p_{10}(\eta)={p_{10}}^{0}(\eta)-\frac{\eta}{\gamma} \int_{0}^{1} p_{10}(\tau) F_{0}(\eta, \tau) d \tau \\
& q_{20}(\eta)=q_{20}{ }^{0}(\eta)-\eta \int_{0}^{1} q_{20}(\tau) G_{10}(\eta, \tau) d \tau
\end{aligned}
$$

where, as before

$$
\begin{aligned}
& p_{1 n}(\zeta)=p_{2 n}(\zeta) \\
& q_{1 n}(\zeta)=-q_{2 n}(\zeta) / \gamma
\end{aligned}
$$

and

$$
\begin{aligned}
s_{1 n}{ }^{0}(\eta) & =\frac{2 \alpha^{2} \beta A i^{n+1}}{\gamma k_{2}{ }^{3}}\left(\frac{\eta}{\alpha}\right)^{1 / 2} J_{n+1 / 2}(\alpha r), \\
p_{1 n}{ }^{0}(\eta) & =\frac{\left[A\left({k_{2}}^{2}-2 \beta^{2}\right) / k_{2}-\beta B\right]}{(\gamma+1)} i^{n+1} \alpha^{1 / 2} \eta J_{n-1 / 2}(\alpha \eta), \\
{q_{1 n}}^{0}(\eta) & =\frac{\left[A\left({k_{2}}^{2}-2 \beta^{2}\right) / k_{2}-\gamma \beta B\right]}{\gamma(\gamma+1)} \alpha^{1 / 2} i^{n+1} \eta J_{n+3 / 2}(\alpha \eta),
\end{aligned}
$$

with the new quantities

$$
\begin{aligned}
& p_{10}{ }^{0}(\eta)=\frac{A}{k_{2} \gamma}\left(k_{2}{ }^{2}-2 \beta^{2}\right) i \eta \alpha^{1 / 2} J_{3 / 2}(\alpha \eta), \\
& q_{20}{ }^{0}(\eta)=-i \alpha^{1 / 2} \beta \eta B J_{3 / 2}(\alpha \eta),
\end{aligned}
$$

and where $F_{1 n}(\eta, \tau), F_{2 n}(\eta, \tau), F_{3 n}(\tau, \eta)$ etc. are defined in (37). Reference should also be made to (23).

As in [1], Eqs. (37) may be converted into integrals over the branch cuts between $\pm k_{1}, \pm k_{2}$, in consequence of the asymptotic behavior of $A(\zeta), B(\zeta)$ and $C(\zeta) \rightarrow 0\left(\zeta^{-1}\right)$ for large $\zeta$. The results of the branch line integrations are in (38) and (44).

The function

$$
G_{0}(\eta, \tau)=\int_{0}^{\infty} C(\zeta) J_{3 / 2}(\zeta \eta) J_{3 / 2}(\zeta \tau) d \zeta
$$

can be obtained from the second part of (37b). In any event, all these kernels are $0\left(k^{2}\right)$ or higher, so Eqs. (35) and (36) may be solved by iteration of the zero ${ }^{\text {th }}$-order terms $s_{1 n}(\zeta), p_{1 n}(\zeta)$, etc.

The results for the cases $n<0$ can be derived from the symmetry relations with the corresponding cases $n>0$. From the integral equations, it can be seen that

$$
S_{-n}(\zeta)=(-1)^{n} S_{n}(\zeta), \quad L_{-n}(\zeta, \beta)=(-1)^{n} L_{n}(\zeta,-\beta), \quad Q_{2 n}(\zeta, \beta)=(-1)^{n} Q_{2 n}(\zeta,-\beta)
$$


4. Effective elastic modulus. Below, we shall consider the problem of the scattering of $S$-waves from a distribution of randomly oriented cracks. We have a choice of considering the scattering by a single crack aligned in the $x-z$ plane as above, for different angles of incidence and polarization, or considering a single direction of propagation and polarization of the incident wave, for different angles of orientation of cracks. We consider the second method of solution here for two reasons: first, this description is the more physically understandable of the two. Second, along the way, we shall be able to obtain the single crack Green's function for scattering in a coordinate system relative to the incident wave, which will be of use in case the orientation of a suite of cracks is not completely isotropic. It should be noted that the question whether the crack or the incident wave gives the more appropriate coordinate system does not arise in the case of incident $P$-waves considered in [1], since the incident field is longitudinally polarized; the complication arises in this case because of the transverse polarization of the incident $S$-wave field.

We describe the incident field in a coordinate system $(X, Y, Z)$ fixed in the elastic body. The incident field can be written as

$$
\mathbf{u}=i \mathbf{c} \times \mathbf{k}_{2} \psi^{0}-\left(\mathbf{c} \times \mathbf{k}_{2}\right) \times \mathbf{k}_{2} \chi^{0}
$$

where $\mathbf{c}$ is an arbitrarily chosen constant vector and $\mathbf{k}_{2}$ is a vector of magnitude equal to the shear wave number and pointing in the direction of propagation. We choose $\chi^{0}=0$, $\mathbf{c}=\mathbf{e}_{X}$ and $\mathbf{k}_{2}=k_{2} \mathbf{e}_{Z}$ so that $\psi_{0}=\Gamma \exp \left(i k_{2} Z\right)$ is the representation of a shear wave traveling in the $Z$-direction and polarized along the $Y$-axis. The strength of the incident potential is $\Gamma$.

We write the incident field in the coordinate system $(x, y, z)$ referred to a circular crack. Let the usual polar angles describing the orientation of the coordinate system $(x, y, z)$ relative to $(X, Y, Z)$ be $\left(\theta_{0}, \phi_{0}\right)$. In the coordinate system $(x, y, z)$ the incident field is

$$
\left(\begin{array}{c}
\chi^{0} \\
\psi^{0}
\end{array}\right)=\left(\begin{array}{c}
A / k_{2} \\
B
\end{array}\right) \exp \left(i\left[\beta z+\alpha r \cos \left(\phi-\phi_{0}\right)\right]\right)
$$

where

$$
\begin{array}{ll}
r=\left(x^{2}+y^{2}\right)^{1 / 2}, & A=-i \Gamma \cos \phi_{0} / \sin \theta_{0}, \\
\beta=k_{2} \cos \theta_{0}, & B=-\Gamma \sin \phi_{0} / \sin \theta_{0} . \\
\alpha=k_{2} \sin \theta_{0}, &
\end{array}
$$

Thus we have obtained the amplitudes of the potentials $\chi^{0}$ and $\psi^{0}$ given in Sec. 2 above. The results of the scattering calculation given in Sec. 3 are obtained in consequence.

An expression for deriving the effective elastic modulus of an aggregate of circular scatterers in the long wavelength limit was derived in [1]. This expression is

$$
\tau_{i j}{ }^{0} L_{1}\left(\tau_{i j}{ }^{0}\right) v={\tau_{i j}}^{0} L_{0}\left(\tau_{i j}{ }^{0}\right) v+\sum_{p=1}^{N}{\tau_{i j}}^{0} \int_{S_{p}} n_{j}\left[u_{i}\right] d s
$$

where $\tau_{i j}{ }^{0}$ is the stress in the incident elastic wave as the frequency $\omega \rightarrow 0$ and $L_{1}$ and $L_{0}$ are the Hookean operators for a homogeneous composite and a homogeneous unflawed medium respectively; $v$ is the volume of space occupied by $N$ of these cracks; $S_{p}$ is the area of the $p^{\text {th }}$ crack; $\left[u_{i}\right]$ is the jump in displacement across the two crack faces. 
The jump in the components of the displacement $\left[u_{r}\right]$ and $\left[u_{\phi}\right]$ in the coordinate system $(x, y, z)$ are found by following the procedure in $(49) ;(r, \phi)$ are circular coordinates in the plane of the crack. We find

$$
\begin{aligned}
& {\left[u_{r}\right]=-2 i \sum_{n=-\infty}^{\infty} \exp (i n \phi)\left\{\int_{0}^{\infty} \zeta L_{n}(\zeta) J_{n}{ }^{\prime}(\zeta r) d \zeta+\frac{n}{r} \int_{0}^{\infty} Q_{2 n}(\zeta) J_{n}(\zeta r) d \zeta\right\},} \\
& {\left[u_{\phi}\right]=2 \sum_{n=-\infty}^{\infty} \exp (\operatorname{in} \phi)\left\{\frac{n}{r} \int_{0}^{\infty} L_{n}(\zeta) J_{n}(\zeta r) d \zeta+\int_{0}^{\infty} \zeta Q_{2 n}(\zeta) J_{n}{ }^{\prime}(\zeta r) d \zeta\right\} .}
\end{aligned}
$$

The dominant terms in expressions (52) and (53) are those for which $n=1$. We use expressions (26), (35a, b), (36a), (30c, d) and the symmetry relations given at the end of Sec. 3. Then Eqs. (52) and (53) become in the case $n=1$

$$
\begin{aligned}
& {\left[u_{r}\right]=\frac{8}{\pi} \frac{\alpha}{\gamma+1}\left(a^{2}-r^{2}\right)^{1 / 2}\left\{\beta B \sin \left(\phi-\phi_{0}\right)+\frac{i A}{k_{2}}\left(k_{2}{ }^{2}-2 \beta^{2}\right) \cos \left(\phi-\phi_{0}\right)\right\},} \\
& {\left[u_{\phi}\right]=\frac{8}{\pi} \frac{\alpha}{\gamma+1}\left(a^{2}-r^{2}\right)^{1 / 2}\left\{\beta B \cos \left(\phi-\phi_{0}\right)-\frac{i A}{k_{2}}\left(k_{2}{ }^{2}-2 \beta^{2}\right) \sin \left(\phi-\phi_{0}\right)\right\} .}
\end{aligned}
$$

The dominant term in expression (49) is that for $m=0$, which gives

$$
\left[u_{z}\right]=\frac{8}{\pi} \frac{\alpha^{2} \beta}{\gamma k_{2}} i A\left(a^{2}-r^{2}\right)^{1 / 2}
$$

where $a$ is the radius of the crack and

$$
\gamma=2\left(1-k_{1}{ }^{2} / k_{2}^{2}\right)
$$

The result of carrying out the integration is

$$
\int \tau_{i j} n_{i}\left[u_{1}\right] d S=\frac{16}{3} \mu_{0} a^{3}\left\{\frac{1}{(\gamma+1)}\left[\alpha^{2} \beta^{2} B^{2}-\frac{\left(k_{2}{ }^{2}-2 \beta^{2}\right)^{2} \alpha^{2} A^{2}}{k_{2}{ }^{2}}\right]-\frac{1}{\gamma k_{2}{ }^{2}} \alpha^{4} \beta^{2} A^{2}\right\}
$$

and the result of the operation with $L_{1}$ is

$$
\tau_{i j}{ }^{0} L_{1}\left(\tau_{i j}{ }^{0}\right)=\mu_{1} k_{2}{ }^{4}\left(\psi^{0}\right)^{2} .
$$

$A$ and $B$ are given above. Thus the effective compliance becomes

$$
\begin{aligned}
\left\langle\frac{1}{\mu_{1}}\right\rangle=\frac{1}{\mu_{0}}\left[1+\sum_{p=1}^{N} \frac{16}{3} \frac{a_{p}{ }^{3}}{v}\left\{\frac{\cos ^{2} \theta_{0 p} \sin ^{2} \phi_{0 p}}{(\gamma+1)}+\frac{\left(1-2 \cos ^{2} \theta_{0 p}\right)^{2} \cos ^{2} \phi_{0 p}}{(\gamma+1)}\right.\right. & \\
& \left.\left.+\frac{1}{\gamma} \sin ^{2} \theta_{0 p} \cos ^{2} \theta_{0 p} \cos ^{2} \phi_{0 p}\right\}\right]
\end{aligned}
$$

where $\left(\theta_{0 p}, \phi_{0 v}\right)$ are the Eulcrian angular coordinates [2] of the normal to the $p$ th crack relative to the direction of incidence and polarization of the field of the excitation.

We can obtain the effective shear modulus for a distribution of cracks which are randomly oriented by integrating over $\left(\theta_{0}, \phi_{0}\right)$ for the case in which all the radii are assumed to be equal, $a_{\rho}=a$. In this case, we get the result

$$
\left\langle\frac{1}{\mu_{1}}\right\rangle=\frac{1}{\mu_{0}}\left[1+\frac{32}{15} \frac{N a^{3}}{v}\left\{\frac{\lambda_{0}+2 \mu_{0}}{3 \lambda_{0}+4 \mu_{0}}+\frac{\lambda_{0}+2 \mu_{0}}{12\left(\lambda_{0}+\mu_{0}\right)}\right\}\right] .
$$

REFERENCE

[1] H. D. Garbin and L. Knopoff, The compressional modulus of a material permeated by a random distribution of circular cracks, Quart. Appl. Math. 30, 453-464 (1973)

[2] H. Goldstein, Classical mechanics, Addison-Wesley, 1953. p. 107 